\title{
Stationary Cylindrical Anisotropic Fluid
}

\author{
F. Debbasch ${ }^{1 *}$ L. Herrera ${ }^{2 \dagger}$ \\ P. R. C. T. Pereira ${ }^{3 \ddagger}$ and N. O. Santos ${ }^{1,4,5 \S}$ \\ ${ }^{1}$ Université Pierre et Marie Curie - CNRS/FRE 2460, \\ LERMA/ERGA, Tour 22-12, 4ème étage, Boîte 142, 4 place Jussieu, \\ 75252 Paris Cedex 05, France. \\ ${ }^{2}$ Escuela de Física, Facultad de Ciencias, \\ Universidad Central de Venezuela, Caracas, Venezuela. \\ ${ }^{3}$ Centro Brasileiro de Pesquisas Físicas, 22290-180 Rio de Janeiro RJ, Brazil. \\ ${ }^{4}$ School of Mathematical Sciences, Queen Mary, \\ University of London, London E1 4NS, UK. \\ ${ }^{5}$ Laboratório Nacional de Computação Científica, 25651-070 Petrópolis RJ, Brazil.
}

November 14, 2018

\begin{abstract}
We present the whole set of equations with regularity and matching conditions required for the description of physically meaningful stationary cylindrically symmmetric distributions of matter, smoothly matched to Lewis vacuum spacetime. A specific example is given. The electric and magnetic parts of the Weyl tensor are calculated, and it is shown that purely electric solutions are necessarily static. Then, it is shown that no conformally flat stationary cylindrical fluid exits, satisfying regularity and matching conditions.
\end{abstract}

\footnotetext{
*e-mail: debbasch@lra.ens.fr

$\dagger$ e-mail: laherrera@movistar.net.ve

†e-mail: terra@cbpf.br

§e-mail: santos@ccr.jussieu.fr and N.O.Santos@qmul.ac.uk
} 


\section{Introduction}

In a recent paper [1] we have presented a systematic study of static cylindrically symmetric matter distributions. To justify such endeavour we mentioned, on the one hand the still ongoing discussion about the precise meaning of the independent parameters of the corresponding vacuum spacetime, and on the other, the renewed interest in cylindrically symmetric sources in relation with different, classical and quantum, aspects of gravitation (see [2] and references therein).

It is clear that the extension of the previous study to the stationary case, is not only justified by the same arguments, but in addition allows for studying the role of the rotation of the source.

However, it should be stressed that in spite of the simplifications introduced by the cylindrical symmetry, a purely analytical handling of the problem requires further restrictions. Accordingly, we shall consider here the shear-free (rigid rotation) case.

As in [1] we want to bring out the relationship between the Weyl tensor and different aspects of the source. This last question is in turn motivated by the very conspicuous link existing in the spherically symmetric case between the Weyl tensor, the inhomogeneity of the energy density and the anisotropy of pressure [3]. For doing so we have calculated the Weyl components as well as the components of its electric and magnetic parts. Unfortunately, no such link can be established in our case. However it is shown that purely electric solutions are necessarily static. This last result, together with results obtained in [1] imply that no conformally flat stationary cylindrical fluid exists, satisfying regularity and matching condition.

The paper is organized as follows: in section 2 we present the general form of the energy momentum tensor, the line element, the Einstein equations, the regularity conditions and the active gravitational mass. The exterior spacetime as well as junction conditions are discussed in section 3. In section 4 a specific example, which we believe is new, is presented. In section 5 the electric and magnetic parts of Weyl tensor are presented and the non existence of conformally flat models satisfying Darmois conditions is established. Finally, some conclusions are presented in the last section and the components of Weyl tensor are given in an appendix. 


\section{Inside the source}

We shall first describe the kind of source we are dealing with as well as its spacetime. Then we shall write the corresponding Einstein equations and discuss the regularity conditions to assure the correct behaviour of the solutions. Finally we present a very compact expression for the active gravitational mass of the source.

\subsection{The source}

We consider a stationary cylindrically symmetric anisotropic non-dissipative fluid bounded by a cylindrical surface $\Sigma$ and with energy momentum tensor given by

$$
T_{\alpha \beta}=\left(\mu+P_{r}\right) V_{\alpha} V_{\beta}+P_{r} g_{\alpha \beta}+\left(P_{\phi}-P_{r}\right) K_{\alpha} K_{\beta}+\left(P_{z}-P_{r}\right) S_{\alpha} S_{\beta},
$$

where, $\mu$ is the energy density, $P_{r}, P_{z}$ and $P_{\phi}$ are the principal stresses and $V_{\alpha}, K_{\alpha}$ and $S_{\alpha}$ are four-vectors satisfying

$$
V^{\alpha} V_{\alpha}=-1, \quad K^{\alpha} K_{\alpha}=S^{\alpha} S_{\alpha}=1, \quad V^{\alpha} K_{\alpha}=V^{\alpha} S_{\alpha}=K^{\alpha} S_{\alpha}=0 .
$$

\subsection{The interior spacetime}

We assume for interior metric to $\Sigma$ the general stationary cylindrically symmetric line element, which can be written

$$
d s^{2}=-f d t^{2}+2 k d t d \phi+e^{\gamma}\left(d r^{2}+d z^{2}\right)+l d \phi^{2},
$$

where $f, k, \gamma$ and $l$ are all functions of $r$. To represent cylindrical symmetry, we impose the following ranges on the coordinates

$$
-\infty \leq t \leq \infty, \quad 0 \leq r, \quad-\infty<z<\infty, \quad 0 \leq \phi \leq 2 \pi
$$

We number the coordinates $x^{0}=t, x^{1}=r, x^{2}=z$ and $x^{3}=\phi$ and we choose the fluid being at rest in this coordinate system, hence from (2) and (3) we have

$$
V_{\alpha}=-\sqrt{f} \delta_{\alpha}^{0}+\frac{k}{\sqrt{f}} \delta_{\alpha}^{3}, \quad S_{\alpha}=e^{\gamma / 2} \delta_{\alpha}^{2}, \quad K_{\alpha}=\left(l+\frac{k^{2}}{f}\right)^{1 / 2} \delta_{\alpha}^{3} .
$$


It is worth noticing that we are assuming that our coordinates are corotating with the fluid $\left(V^{\alpha}=\delta_{0}^{\alpha} / \sqrt{f}\right)$, which implies, as it can be very easily shown, that the fluid is shear-free. Thus we shall consider a shear-free, expansionless fluid distribution.

\subsection{The Einstein equations}

For the Einstein field equations, $G_{\alpha \beta}=\kappa T_{\alpha \beta}$, with (1), (3) and (5) we have the non-null components

$$
\begin{array}{r}
G_{00}=-\frac{1}{4 D^{2} e^{\gamma}}\left[2 f D^{2} \gamma^{\prime \prime}+4 f D D^{\prime \prime}-2 D^{2} f^{\prime \prime}+2 D D^{\prime} f^{\prime}\right. \\
\left.-3 f\left(f^{\prime} l^{\prime}+k^{2}\right)\right]=\kappa \mu f, \\
G_{03}=\frac{1}{4 D^{2} e^{\gamma}}\left[2 k D^{2} \gamma^{\prime \prime}+4 k D D^{\prime \prime}-2 D^{2} k^{\prime \prime}+2 D D^{\prime} k^{\prime}\right. \\
\left.-3 k\left(f^{\prime} l^{\prime}+k^{\prime 2}\right)\right]=-\kappa \mu k, \\
G_{11}=\frac{1}{4 D^{2}}\left(2 D D^{\prime} \gamma^{\prime}+f^{\prime} l^{\prime}+k^{2}\right)=\kappa P_{r} e^{\gamma}, \\
G_{22}=-\frac{1}{4 D^{2}}\left(2 D D^{\prime} \gamma^{\prime}-4 D D^{\prime \prime}+f^{\prime} l^{\prime}+k^{\prime 2}\right)=\kappa P_{z} e^{\gamma}, \\
G_{33}=\frac{1}{4 D^{2} e^{\gamma}}\left[2 l D^{2} \gamma^{\prime \prime}+4 l D D^{\prime \prime}-2 D^{2} l^{\prime \prime}+2 D D^{\prime} l^{\prime}\right. \\
\left.-3 l\left(f^{\prime} l^{\prime}+k^{2}\right)\right]=\frac{\kappa}{f}\left(\mu k^{2}+P_{\phi} D^{2}\right) .
\end{array}
$$

where the primes stand for differentiation with respect to $r$ and

$$
D^{2}=f l+k^{2}
$$

Thus we have five equations for the eight unknown functions, namely $f$, $k, \gamma, l, \mu, P_{r}, P_{z}$ and $P_{\phi}$. Therefore three equations of state relating the matter variables or ad hoc assumptions on the metric functions have to be imposed in order to integrate (6)-(10) (e.g. see the example in section 4 below).

The Bianchi identity $T_{r ; \beta}^{\beta}=0$ from (1) reduces to,

$$
P_{r}^{\prime}+\frac{1}{2}\left(\mu+P_{r}\right) \frac{f^{\prime}}{f}+\left(P_{r}-P_{\phi}\right) \frac{D^{\prime}}{D}+\frac{1}{2}\left(P_{r}-P_{z}\right) \gamma^{\prime}=0 .
$$


From (6) and (7) we have

$$
k G_{00}+f G_{03}=\frac{D}{2 e^{\gamma}}\left(\frac{k f^{\prime}-f k^{\prime}}{D}\right)^{\prime}=0,
$$

which allows to be integrated, producing

$$
k f^{\prime}-f k^{\prime}=c_{1} D
$$

where $c_{1}$ is an integration constant.

\subsection{Regularity conditions}

Regularity conditions on the symmetry axis imply [4]

$$
\lim _{r \rightarrow 0} \frac{g^{\alpha \beta} X_{, \alpha} X_{, \beta}}{4 X}=1
$$

with $X=g_{\phi \phi}$. Then (15) yields

$$
\lim _{r \rightarrow 0} \frac{e^{-\gamma} l^{\prime 2}}{4 l}=1
$$

The requirement that $g_{\phi \phi}=0$ on the axis, gives

$$
l \stackrel{0}{=} 0
$$

where $\stackrel{0}{=}$ means that the equation is evaluated at $r=0$.

Since along the axis physically there cannot be singularities, we impose that spacetime tends near the axis to a flat spacetime, hence we scale the coordinates so that as $r \rightarrow 0$ we have

$$
d s^{2}=-d t^{2}+2 \omega r^{2} d t d \phi+d r^{2}+d z^{2}+r^{2} d \phi^{2},
$$

from which it follows that

$$
f \stackrel{0}{=} 1, \quad k \stackrel{0}{=} \gamma \stackrel{0}{=} 0
$$

implying

$$
D \stackrel{0}{=} 0
$$


and from (16) and (18)

$$
l^{\prime} \stackrel{0}{=} 0 .
$$

Finally, from the above and the requirement that Einstein tensor components in (6-10) do not diverge, it follows that

$$
f^{\prime} \stackrel{0}{=} k^{\prime} \stackrel{0}{=} k^{\prime \prime}-k^{\prime} \frac{D^{\prime}}{D} \stackrel{0}{=} 0 .
$$

\subsection{The Whittaker mass}

The Whittaker formula [5] for the active mass per unit length $m$ of a stationary distribution of perfect fluid with energy density $\mu$ and principal stresses $P_{r}, P_{z}$ and $P_{\phi}$ inside a cylinder of surface $\Sigma$ is

$$
m=2 \pi \int_{0}^{r_{\Sigma}}\left(\mu+P_{r}+P_{z}+P_{\phi}\right) \sqrt{-g} d r,
$$

where $g$ is the determinant of the metric. Now substituting (3) and (6-10) we obtain

$$
m=\frac{2 \pi}{\kappa} \int_{0}^{r_{\Sigma}}\left(\frac{l f^{\prime}+k k^{\prime}}{D}\right)^{\prime} d r=\frac{2 \pi}{\kappa} \int_{0}^{r_{\Sigma}}\left(\frac{D f^{\prime}-c_{1} k}{f}\right)^{\prime} d r,
$$

where we have used (14). Integrating (24) and using the regularity conditions on the axis we obtain

$$
m \stackrel{\Sigma}{=} \frac{2 \pi}{\kappa} \frac{D f^{\prime}-c_{1} k^{\prime}}{f} .
$$

\section{$3 \quad$ Exterior spacetime and junction conditions}

For the exterior spacetime of the cylindrical surface $\Sigma$, since the system is stationary, we take the Lewis metric [6] and consider its Weyl class [7],

$$
d s^{2}=-F d t^{2}+2 K d t d \phi+e^{\Gamma}\left(d R^{2}+d z^{2}\right)+L d \phi^{2},
$$

where

$$
\begin{array}{r}
F=a R^{1-n}-a \delta^{2} R^{1+n}, \\
K=-(1-a b \delta) \delta R^{1+n}-a b R^{1-n}, \\
e^{\Gamma}=R^{\left(n^{2}-1\right) / 2}, \\
L=\frac{(1-a b \delta)^{2}}{a} R^{1+n}-a b^{2} R^{1-n},
\end{array}
$$


with

$$
\delta=\frac{c}{a n}
$$

and $a, b, c$ and $n$ are real constants. The coordinates $t, z$ and $\phi$ can be taken as in (3) and with the same ranges (4). The radial coordinates in (3) and (26), $r$ and $R$, are not necessarily continuous on $\Sigma$ in order to preserve the range of $\phi$ in (4). Also, since we are considering an expansionless cylinder and the fluid is at rest in our coordinate system, the equation of the boundary surface should read $r \stackrel{\Sigma}{=}$ constant and $R \stackrel{\Sigma}{=}$ constant, from inside and outside, respectively.

In accordance with Darmois junction conditions [8] we obtain that the metric coefficients (3) and (26) and its derivatives must be continuous across $\Sigma$,

$$
\begin{array}{r}
f \stackrel{\Sigma}{=} F, \quad k \stackrel{\Sigma}{=} K, \quad \gamma \stackrel{\Sigma}{=} \Gamma, \quad l \stackrel{\Sigma}{=} L, \\
\frac{f^{\prime}}{f} \stackrel{\Sigma}{=} \frac{1}{R}+n \frac{\delta^{2} R^{n}+R^{-n}}{\delta^{2} R^{1+n}-R^{1-n}}, \\
\frac{k^{\prime}}{k} \stackrel{\Sigma}{=} \frac{1}{R}+n \frac{(1-a b \delta) \delta R^{n}-a b R^{-n}}{(1-a b \delta) \delta R^{1+n}+a b R^{1-n}}, \\
\gamma^{\prime} \stackrel{\Sigma}{=} \frac{n^{2}-1}{2 R}, \\
\frac{l^{\prime}}{l} \stackrel{\Sigma}{=} \frac{1}{R}+n \frac{(1-a b \delta)^{2} R^{n}+a^{2} b^{2} R^{-n}}{(1-a b \delta)^{2} R^{1+n}-a^{2} b^{2} R^{1-n}} .
\end{array}
$$

hence we have from (8) $P_{r} \stackrel{\Sigma}{=} 0$, as expected, and for (14) and (25)

$$
\begin{array}{r}
c_{1}=2 c \\
m=\sigma+\frac{b c}{2},
\end{array}
$$

where $\sigma$ is the Newtonian mass per unit length, as measured outside the source, given by

$$
\sigma=\frac{1-n}{4}
$$

The two parameters $b$ and $c$ are responsible for the stationarity of the spacetime, accordingly the second term in (38) may be interpreted as the "rotational" contribution to the active gravitational mass of the source. 
For a cylindrically symmetric rigidly rotating dust, van Stockum solution [9], one obtains

$$
b c=-\frac{(1-n)^{2}}{4}
$$

or with $(38-40)$

$$
m=\sigma(1-2 \sigma),
$$

which could be interpreted as if, in this case, rotation diminishes the active gravitational mass. At first sight this result seems counterintuitive, as we should expect the energy of the rotational motion to increase the total energy of the source.

However, the situation is not so simple. First of all observe that the van Stockum solution has no static limit (see next section). Indeed, reducing its rotation to zero amounts to delete the source (the spacetime becomes flat). Therefore it is not clear that in this case, $m$ as given by (38) might be interpreted as describing the static plus the "rotational" contributions to the active gravitational mass of the source, since no static contribution exists for this case.

In the same line of arguments it is worth noticing that circular geodesics of test particles become null when $\sigma=1 / 4$, for both, the static and stationary cylinder [10], but this corresponds to $m=1 / 4$ in the first case and $m=1 / 8$ in the van Stockum case.

On the other hand, we know of at least one example where the angular momentum of the source diminishes the effective active gravitational mass of the source: the Kerr metric. Indeed, in this later case the equation for radial geodesic on the symmetry axis reads:

$$
\ddot{r}=-M \frac{r^{2}-a^{2}}{\left(r^{2}+a^{2}\right)^{2}}
$$

where the dot stands for differentiation with respect to the proper time, $r$ is the radial Boyer-Lindquist coordinate and $a$ and $M$ are the two parameters of the Kerr metric. It is evident from (42) that the angular momentum of the source $a$ diminishes the attraction force on the test particle, as compared with the static case.

All this having been said, we do not know at this point whether or not some kind of mechanism similar to (or different from) the one existing in the Kerr spacetime is acting in the van Stockum case, and more important we do not know what the physics underlying such mechanism, might be. 


\section{An interior solution}

In order to illustrate the use of all the equations above, let us present a simple model which we believe is new. As we shall see, even in a very restrictive situation, as the one presented below, a purely analytical treatment of the problem is not suitable, and one should appeal to numerical methods, for a complete description of the solution. Nevertheless, some information about the properties of the model may be obtained from the analytical approach.

Thus, let us assume for simplicity the equations of state

$$
P_{z}=\alpha \mu, \quad P_{r}=P_{\phi}=0
$$

with $\alpha=$ constant. When $\alpha=0$ (43) reduces to the van Stockum solution

[9] for a cylindrically symmetric rotating dust which is given by

$$
\begin{array}{r}
f=1, \quad k=\omega r^{2}, \quad \gamma=-\omega^{2} r^{2}, \quad l=r^{2}\left(1-\omega^{2} r^{2}\right), \\
\kappa \mu=4 \omega^{2} e^{\omega^{2} r^{2}},
\end{array}
$$

where $\omega$ is a constant.

Substituting (43) into (12) we have for $\mu \neq 0$

$$
\alpha \gamma^{\prime}=\frac{f^{\prime}}{f},
$$

and integrating we obtain

$$
e^{\alpha \gamma}=c_{2} f
$$

where $c_{2}$ is an integration constant. Considering the regularity conditions (19) we can rewrite (47) as

$$
e^{\alpha \gamma}=f
$$

The field equations (6-10) with (43) reduce to

$$
\begin{aligned}
2 D D^{\prime} \gamma^{\prime}+f^{\prime} l^{\prime}+k^{2} & =0 \\
4(1+\alpha) f D D^{\prime \prime}+\alpha\left[2 f D^{2} \gamma^{\prime \prime}-2 D^{2} f^{\prime \prime}+2 D D^{\prime} f^{\prime}-3 f\left(f^{\prime} l^{\prime}+k^{2}\right)\right] & =0
\end{aligned}
$$

Substituting (14), (37) and (46) into (49) and considering (11) we have

$$
2(1+\alpha) f f^{\prime} D^{\prime}-\alpha D\left(f^{\prime 2}-4 c^{2}\right)=0
$$


while substituting (46) and (49) into (50) we obtain

$$
2(1+\alpha) f^{2} D^{\prime \prime}+(1-\alpha) D f f^{\prime \prime}-D f^{\prime 2}+(3+\alpha) f f^{\prime} D^{\prime}=0 .
$$

Now from (51) and (52) we have

$$
\left(f^{\prime 2}+4 \alpha c^{2}\right)\left[2(1+\alpha) f f^{\prime \prime}-(2+\alpha) f^{\prime 2}+4 \alpha c^{2}\right]=0 .
$$

If $\alpha=0$ then (53) reduces to $f^{\prime}=0$, giving the van Stockum's solution (44). However if $\alpha \neq 0$ and since

$$
f^{\prime 2}+4 \alpha c^{2} \neq 0
$$

considering the transformation

$$
f^{\prime 2}=x \neq 0
$$

(53) reduces to

$$
\frac{f^{\prime}}{f}=\frac{(1+\alpha) x^{\prime}}{(2+\alpha) x-4 \alpha c^{2}} .
$$

Integrating (56) we obtain

$$
f=c_{3}\left[(2+\alpha) x-4 \alpha c^{2}\right]^{(1+\alpha) /(2+\alpha)},
$$

where $c_{3}$ is an integration constant. From the regularity conditions (19) and (22) we can rewrite (57),

$$
f=\left(1-\frac{2+\alpha}{4 \alpha c^{2}} x\right)^{(1+\alpha) /(2+\alpha)},
$$

or with the aid of (46) it becomes

$$
f=\left[1-\frac{(2+\alpha) \alpha}{4 c^{2}} f^{2} \gamma^{\prime 2}\right]^{(1+\alpha) /(2+\alpha)}
$$

From (59) we see that when $\alpha=0$ it reduces to the van Stockum solution (44).

Feeding back (55) into (58) we could in principle integrate to obtain $f=f(r)$, unfortunately though, the integral can only be expressed in terms 
of hypergeometric functions, therefore further specification of the solution is only possible numerically.

However, some general properties of the solution may be found without performing the integration of (58).

Thus, the matching of (46) or (51) using (32-36) produces

$$
\alpha \stackrel{\Sigma}{=} \frac{2}{n^{2}-1}\left(1+n \frac{\delta^{2} R^{n}+R^{-n}}{\delta^{2} R^{n}-R^{-n}}\right),
$$

showing that $\alpha$ can have positive as well as negative values for different parameters that describe the source.

Substituting (46), (49-51) and (58) into (6) we obtain

$$
\kappa \mu e^{\gamma}=\left[\frac{2 c}{(1+\alpha) f}\right]^{2} .
$$

At the axis $r=0$ (61) becomes

$$
\kappa \mu \stackrel{0}{=}\left(\frac{2 c}{1+\alpha}\right)^{2}
$$

If we fix the stationary parameter $c$ we observe from (62) that if $\alpha>0$, or $P_{z}>0$, the density $\mu$ diminishes as compared to the van Stockum dust solution; however if $\alpha<0$, or $P_{z}<0$, then $\mu$ increases as compared to the same solution.

The model cannot be specified further until (58) is integrated and the full set of boundary conditions are used. However, as mentioned before this requires the introduction of numerical procedures, which is out of the scope of the paper.

\section{The electric and the magnetic parts of Weyl tensor}

The electric and magnetic parts of Weyl tensor, $E_{\alpha \beta}$ and $H_{\alpha \beta}$, respectively, are formed from the Weyl tensor $C_{\alpha \beta \gamma \delta}$ and its dual $\tilde{C}_{\alpha \beta \gamma \delta}$ by contraction with the four velocity vector $V^{\alpha}$, thus

$$
E_{\alpha \beta}=C_{\alpha \gamma \beta \delta} V^{\gamma} V^{\delta}
$$




$$
H_{\alpha \beta}=\tilde{C}_{\alpha \gamma \beta \delta} V^{\gamma} V^{\delta}=\frac{1}{2} \epsilon_{\alpha \gamma \epsilon \delta} C_{\beta \rho}^{\epsilon \delta} V^{\gamma} V^{\rho}, \quad \epsilon_{\alpha \beta \gamma \delta} \equiv \sqrt{-g} \eta_{\alpha \beta \gamma \delta}
$$

where $\eta_{\alpha \beta \gamma \delta}=+1$ or -1 for $\alpha, \beta, \gamma, \delta$ in even or odd order, respectively, and 0 otherwise, and with

$$
\begin{aligned}
V^{\alpha} & =\frac{\delta_{0}^{\alpha}}{\sqrt{f}}, \\
\sqrt{-g} & =D e^{\gamma} .
\end{aligned}
$$

Then it follows that there are only three non-vanishing electric components, namely

$$
\begin{aligned}
& E_{11}=\frac{C_{0101}}{f}, \\
& E_{22}=\frac{C_{0202}}{f}, \\
& E_{33}=\frac{C_{0303}}{f},
\end{aligned}
$$

however they are not independent, since, by virtue of (85) they satisfy the relation

$$
E_{11}+E_{22}=-\frac{f e^{\gamma}}{D^{2}} E_{33}
$$

Thus, there are two independent electric components of the Weyl tensor.

On the other hand it can be easily checked that there is only one independent magnetic component, namely

$$
H_{12}=\frac{1}{D}\left(C_{0223}-\frac{k}{f} C_{0202}\right) .
$$

Accordingly, if we demand our spacetime to be purely electric $\left(H_{\alpha \beta}=0\right)$, then using (14), (71), (79) and (82) we obtain

$$
\gamma^{\prime}\left(k f^{\prime}-f k^{\prime}\right)=0
$$

implying that either $\gamma^{\prime}=0$ or $k f^{\prime}-f k^{\prime}=0$. In the first case it follows from the regularity conditions that $\gamma=0$. Then matching condition (35) implies $n=1$, however this last condition implies because of (39) that $\sigma=0$, which produces a Minkowski spacetime outside the cylinder. 
On the other hand $k f^{\prime}-f k^{\prime}=0$ implies, because of regularity conditions, that $k=0$.

Thus purely electric cylindrically symmetric spacetimes are necessarily static. This is in agreement with the result by Glass [11] indicating that a necessary and sufficient condition for a shear-free perfect fluid to be irrotational is that the Weyl tensor be purely electric. However, our result does not require the pressure to be isotropic.

Considering the field equations (6), (7), (9) and (10) we can rewrite (67), (68) and (71) as

$$
\begin{array}{r}
E_{11}=\frac{1}{12}\left[\kappa e^{\gamma}\left(7 \mu+5 P_{z}+5 P_{\phi}\right)+\left(4 \frac{D^{\prime}}{D}-3 \frac{f^{\prime}}{f}\right) \gamma^{\prime}-\frac{f^{\prime} l^{\prime}+k^{\prime 2}}{D^{2}}\right], \\
E_{22}=\frac{1}{12}\left[\kappa e^{\gamma}\left(\mu+5 P_{z}-P_{\phi}\right)-\left(2 \frac{D^{\prime}}{D}-3 \frac{f^{\prime}}{f}\right) \gamma^{\prime}-\frac{f^{\prime} l^{\prime}+k^{\prime 2}}{D^{2}}\right], \\
H_{12}=\frac{1}{4 D}\left(k^{\prime}-\frac{f^{\prime}}{f} k\right) \gamma^{\prime},
\end{array}
$$

and with (14) we have

$$
H_{12}=-\frac{c_{1}}{4} \frac{\gamma^{\prime}}{f}
$$

or using (37)

$$
H_{12}=-\frac{c}{2} \frac{\gamma^{\prime}}{f}
$$

Since the parameter $c$ can be shown to be proportional to the vorticity of the source [7], this last equation reinforces further the belief that rotation is one of the sources of the magnetic part of Weyl tensor [12]. However, it should be noticed that spacetimes are known which have vorticity but zero $H_{\alpha \beta}$ [13]. The most conspicuous example of this kind of spacetime being the Gödel solution [14].

\section{Conclusions}

We have deployed the equations describing the stationary (shear-free) cylinder, as well as the regularity and matching conditions and the active gravitational mass. Then a simple model has been presented to illustrate the way

in which models can be found. 
Next, we have calculated the Weyl tensor components, as well as its electric and magnetic parts. It has been established that purely electric solutions are necessarily static. This result, together with a previously known result [1] about the nonexistence of (static) conformally flat solution which satisfies regularity conditions and matches smoothly to Levi-Civita spacetime on the boundary surface, implies the nonexistence of interior (stationary) conformally flat solution which satisfies regularity conditions and matches smoothly to Lewis spacetime on the boundary surface.

Finally, a link between the magnetic part of the Weyl tensor and a parameter related to the vorticity of the source has been found.

\section{Acknowledgments}

NOS gratefully acknowledges financial assistance from EPSRC United Kingdom and CNPq Brazil.

\section{Appendix}

The spacetime (3) has the following non-null components of the Weyl tensor $C_{\alpha \beta \gamma \delta}$,

$$
\begin{array}{r}
C_{0101}=-\frac{1}{12}\left[f \gamma^{\prime \prime}-3\left(f \frac{D^{\prime}}{D}-f^{\prime}\right) \gamma^{\prime}+2 f \frac{D^{\prime \prime}}{D}\right. \\
\left.-3 f^{\prime \prime}+3 \frac{D^{\prime}}{D} f^{\prime}-2 \frac{f}{D^{2}}\left(f^{\prime} l^{\prime}+k^{2}\right)\right], \\
C_{0202}=-\frac{1}{12}\left[f \gamma^{\prime \prime}+3\left(f \frac{D^{\prime}}{D}-f^{\prime}\right) \gamma^{\prime}-4 f \frac{D^{\prime \prime}}{D}\right. \\
\left.+3 f^{\prime \prime}-3 \frac{D^{\prime}}{D} f^{\prime}+4 \frac{f}{D^{2}}\left(f^{\prime} l^{\prime}+k^{\prime 2}\right)\right], \\
C_{0303=-D^{2} e^{-2 \gamma} C_{1212}=} \frac{D^{2} e^{-\gamma}}{6}\left[\gamma^{\prime \prime}-\frac{D^{\prime \prime}}{D}+\frac{1}{D^{2}}\left(f^{\prime} l^{\prime}+k^{\prime 2}\right)\right], \\
C_{0113}=-\frac{1}{12}\left[k \gamma^{\prime \prime}-3\left(k \frac{D^{\prime}}{D}-k^{\prime}\right) \gamma^{\prime}+2 k \frac{D^{\prime \prime}}{D}\right.
\end{array}
$$




$$
\begin{aligned}
- & \left.3 k^{\prime \prime}+3 \frac{D^{\prime}}{D} k^{\prime}-2 \frac{k}{D^{2}}\left(f^{\prime} l^{\prime}+k^{2}\right)\right], \\
C_{0223}=-\frac{1}{12} & {\left[k \gamma^{\prime \prime}+3\left(k \frac{D^{\prime}}{D}-k^{\prime}\right) \gamma^{\prime}-4 k \frac{D^{\prime \prime}}{D}\right.} \\
+ & \left.3 k^{\prime \prime}-3 \frac{D^{\prime}}{D} k^{\prime}+4 \frac{k}{D^{2}}\left(f^{\prime} l^{\prime}+k^{\prime 2}\right)\right], \\
C_{1313}= & \frac{1}{12}\left[l \gamma^{\prime \prime}-3\left(l \frac{D^{\prime}}{D}-l^{\prime}\right) \gamma^{\prime}+2 l \frac{D^{\prime \prime}}{D}\right. \\
& \left.-3 l^{\prime \prime}+3 \frac{D^{\prime}}{D} l^{\prime}-2 \frac{l}{D^{2}}\left(f^{\prime} l^{\prime}+k^{\prime 2}\right)\right] \\
C_{2323}= & \frac{1}{12}\left[l \gamma^{\prime \prime}+3\left(l \frac{D^{\prime}}{D}-l^{\prime}\right) \gamma^{\prime}-4 l \frac{D^{\prime \prime}}{D}\right. \\
& \left.+3 l^{\prime \prime}-3 \frac{D^{\prime}}{D} l^{\prime}+4 \frac{l}{D^{2}}\left(f^{\prime} l^{\prime}+k^{\prime 2}\right)\right],
\end{aligned}
$$

and they satisfy the relations,

$$
\begin{array}{r}
C_{0101}+C_{0202}=-\frac{f e^{\gamma}}{D^{2}} C_{0303}, \\
C_{0113}+C_{0223}=-\frac{k e^{\gamma}}{D^{2}} C_{0303}, \\
C_{1313}+C_{2323}=\frac{l e^{\gamma}}{D^{2}} C_{0303}, \\
l C_{0101}+f C_{2323}=k\left(C_{0223}-C_{0113}\right),
\end{array}
$$

hence we have three independent Weyl tensor components, which is one more than the number in the static case [1].

\section{References}

[1] Herrera L, Le Denmat G, Marcilhacy G and Santos N O 2005 Int. J. Mod. Phys. D 14657

[2] Evans A 1977 J. Phys. A 10 1303; Kramer D 1988 Class. Quantum Grav. 5 193; Bondi H 1990 Proc. R. Soc. London A 427 259; Davidson W 1990 Gen. Rel. Grav. 22 553; Davidson W 1990 J. Math. Phys. 31 
1972; Bonnor W B 1979 J. Phys. A 12 847; da Silva M F A, Herrera L, Paiva F M and Santos N O 1995 J. Math. Phys. 36 3625; Haggag S and Desokey F 1996 Class. Quantum Grav. 13 3221; Haggag S and Mahmoud R 1998 Il Nuovo Cimento 113B 1131; MacCallum M A H 1998 Gen. Rel. Grav. 30 131; Bonnor B W 1999 The static cylinder in general relativity, in On Einstein's Path ed. Alex Harvey (New York: Springer-Verlag) page 113; Philbin T. 1997 Class. Quantum Grav. 13 1217; Banerjee A, Chaterjee S and Ghosh T 2000 Pramana J. Phys. 54 337; Herrera L, Ruifernández J and Santos N O 2001 Gen. Rel. Grav. 33 515; Herrera L, Santos N O, Teixeira A F F and Wang A Z 2001 Class. Quantum Grav. 18 3847; Ali R 2001 Il Nuovo Cimento 116B 1005; Goncalves S gr-qc/0202042; Bicak J and Zofak M 2002 Class. Quantum Grav. 19 3653; Camci U 2003 Int. J. Mod. Phys. D 12 1431; Arik M and Delice O 2003 Int. J. Mod. Phys. D 12 1095; Qadir A, Saifullah K and Ziad M 2003 Gen. Rel. Grav. 35 1927; Krisch J 2003 Class. Quantum Grav. 20 1605; Konkowski D, Heliwell T and Wieland C 2004 Class. Quantum Grav. 21 265; Gersl J, Keplac P and Horsky J 2004 Gen. Rel. Grav. 36 1399; Hennig J and Neugebauer G gr-qc/0409096; Delice O gr-qc/0411011; Delice O gr-qc/0405065; Bonnor W B 2005 Class. Quantum Grav. 22 803; Salti M and Havare A 2005 Mod. Phys. Lett. A 20 451; Konkowski D and Heliwell T 2006 Gen. Rel. Grav. 381069

[3] Herrera L, Di Prisco A, Martin J, Ospino J, Santos N O and Troconis O 2004 Phys. Rev. D 69084026

[4] Stephani H, Kramer D, MacCallum M, Honselaers C and Herlt E 2003 Exact Solutions to Einstein's Field Equations. Second Edition, (Cambridge University Press, Cambridge)

[5] Whittaker E T 1935 Proc. Roy. Soc. A 149384

[6] Lewis T 1932 Proc. Roy. Soc. 136176

[7] da Silva M F A, Herrera L, Paiva F M and Santos N O 1995 Gen. Rel. Grav. $\mathbf{2 7} 859$

[8] Darmois G 1927 Mémorial des Sciences Mathematiques (Paris: Gauthier-Villars) fasc. 25 
[9] van Stockum W J 1937 Proc. R. Soc. Edinburgh 57135

[10] Herrera L and Santos N O 1988 J. Math. Phys. 393817

[11] Glass E N 1975 J. Math. Phys. 162361

[12] Bonnor W B 1995 Class. Quantum Grav. 121483

[13] Collins C B 1984 J. Math. Phys. 25 995; Sklavenites D 1985 J. Math. Phys. 262279

[14] Maartens R and Basset B 1998 Class. Quantum Grav. 15705 